QUARTERLY OF APPLIED MATHEMATICS

VOLUME LXIV, NUMBER 3

SEPTEMBER 2006, PAGES 483-498

S $0033-569 X(06) 01016-0$

Article electronically published on June 14, 2006

\title{
COMPETITIVE SYSTEMS WITH MIGRATION AND THE POINCARÉ-BENDIXSON THEOREM FOR A 4-DIMENSIONAL CASE
}

\author{
BY \\ JIFA JIANG (Department of Applied Mathematics, Tongji University, Shanghai 200092, People's \\ Republic of China) \\ AND \\ XING LIANG (Department of Mathematics, University of Science and Technology of China, Hefei, \\ Anhui 230026, People's Republic of China)
}

\begin{abstract}
In this paper, dynamics of the $n$-species competitive system with migration is studied. It is proved that if the Jacobian matrix of the system is irreducible at every point in Int $\mathbb{R}_{+}^{2 n}$, then there is a defined countable family of invariant $(2 n-1)$-cells which attract all nonconvergent persistent trajectories. Moreover, it is proved that the Poincaré-Bendixson theorem holds for 2-species competitive systems with migration.
\end{abstract}

1. Introduction. Monotone and competitive dynamical systems have been extensively investigated after a remarkable series of papers by Hirsch (see [1, 2, 3]). A system of ordinary differential equations on the domain $\Omega \subset \mathbb{R}^{m}$ is called a monotone or cooperative system if the off-diagonal elements of the Jacobian matrix of the vector field at every point $x \in \Omega$ are nonnegative, and it is called a competitive system if the offdiagonal elements of the Jacobian matrix at every point $x \in \Omega$ are nonpositive. Smale [11] showed that any vector field on the standard $(m-1)$-dimensional simplex in $\mathbb{R}_{+}^{m}$ can be imbedded in a smooth competitive Kolmogorov vector field

$$
\dot{x}_{i}=F_{i}(x)=x_{i} f_{i}(x), \quad 1 \leq i \leq m, \quad x_{i} \geq 0,
$$

on $\mathbb{R}_{+}^{m}$ for which the simplex is an attractor. On the positive side, in papers [1, 2, 3 ] Hirsch established that the limit sets of competitive systems can be no more complicated than those of general systems in one fewer dimension. Precisely, under the conditions of competition, irreducibility, and dissipation, Hirsch $[3$ proved that for the competitive

Received October 19, 2005.

2000 Mathematics Subject Classification. Primary 34C12, 37N25; Secondary 92D40.

Key words and phrases. Competitive systems with migration, Poincaré-Bendixson theorem. Research of the first author supported by the National Natural Science Foundation of China.

Research of the second author supported by the National Natural Science Foundation of China grant 10401032

E-mail address: jiangjf@mail.tongji.edu.cn

E-mail address: xliang@ustc.edu.cn 
Kolmogorov system there is a canonically defined countable (generically finite) family of disjoint invariant $(m-1)$-cells which attract all nonconvergent persistent trajectories, where a trajectory is said to be persistent if its omega limit set is included in $\operatorname{Int} \mathbb{R}_{+}^{m}=$ $\left\{x=\left(x_{1}, \cdots, x_{m}\right) \in \mathbb{R}^{m}, x_{i}>0, i=1,2, \cdots, m\right\}$. In particular, if the origin is a repeller and the community matrices are strictly negative, then there is a unique closed invariant $(m-1)$-cell attracting all nontrivial trajectories. In this sense, we say the dynamics of competitive systems is 1-codimensional. Wang and Jiang [21] and 22] have verified that the same results hold for the periodic Kolmogorov competitive systems via Poincaré mappings.

Besides, the series of papers by Hirsch are especially important for introducing some powerful techniques for treating monotone flows. Smith [13] developed Hirsch's idea and studied the so-called type-K monotone systems. A system of differential equations $\dot{x}=f(x)$ on $\mathbb{R}_{+}^{m}$ is called a type-K monotone system if the Jacobian $\mathrm{D} f(x)$ of $f$ is type-K monotone at any $x \in \mathbb{R}_{+}^{m}$; that is, there is some $k$ with $1 \leq k \leq m$ such that $\mathrm{D} f(x)$ has the form

$$
\left(\begin{array}{rr}
A_{1} & -A_{2} \\
-A_{3} & A_{4}
\end{array}\right)
$$

in which $A_{1}$ is a $k \times k$ matrix, $A_{2}$ is a $k \times(m-k)$ matrix, $A_{3}$ is an $(m-k) \times k$ matrix, $A_{4}$ is an $(m-k) \times(m-k)$ matrix, each off-diagonal element of $A_{1}$ and $A_{4}$ is nonnegative, and $A_{2}$ and $A_{3}$ are nonnegative matrices. Smith showed that the flow $\varphi_{t}(x)$ generated by the type- $\mathrm{K}$ monotone system is type- $\mathrm{K}$ monotone corresponding to the cone $K=\left\{x \in \mathbb{R}^{m}: x_{i} \geq 0\right.$ for $1 \leq i \leq k$ and $x_{j} \leq 0$ for $\left.k+1 \leq j \leq m\right\}$; that is, if $x, y \in \mathbb{R}_{+}^{m}$ with $x_{i} \leq y_{i}$ for $1 \leq i \leq k$ and $x_{j} \geq y_{j}$ for $k+1 \leq j \leq m$, then for any $t>0$, $\left(\varphi_{t}(x)\right)_{i} \leq\left(\varphi_{t}(y)\right)_{i}$ for $1 \leq i \leq k$ and $\left(\varphi_{t}(x)\right)_{j} \geq\left(\varphi_{t}(y)\right)_{j}$ for $k+1 \leq j \leq m$. For type-K monotone Kolmogorov systems he gave the sufficient conditions for the permanence of the systems, where permanence means there is a compact invariant set $S \subset \operatorname{Int} \mathbb{R}_{+}^{m}$ which attracts all orbits in Int $\mathbb{R}_{+}^{m}$. Afterwards, many researchers continued to investigate the type-K monotone dynamical systems. We refer to Hsu et al. 4, Smith and Thieme [14, Tu and Jiang [19, 18, 20, and Liang and Jiang [6, 7, 8, .

The system $\dot{x}=f(x)$ on $\Omega \subset \mathbb{R}^{m}$ is called a type-K competitive system if $-\mathrm{D} f(x)$ is a type-K monotone matrix at any $x \in \Omega$. Many systems, such as the Field-Noyes models of Belousov-Zhabotinski reaction, due to Murray [10, and all kinds of competitorcompetitor-mutualist models (see 9 and references therein) are type-K competitive Kolmogorov systems according to our definition. We can easily see that the system obtained from the type-K competitive system by time reversal is type-K monotone, and hence the flow generated by the type-K competitive system is backward type-K monotone.

In our papers [7, 9], we considered type-K monotone and type-K competitive Kolmogorov systems, respectively. We showed that many conclusions in Hirsch 3 ] for competitive Kolmogorov systems also hold for type-K monotone and type-K competitive Kolmogorov systems. We proved the existence of the invariant $(m-1)$-manifolds. Moreover, for type-K competitive Kolmogorov systems we proved that all persistent trajectories not converging are attracted by countably many invariant $(m-1)$-manifolds, and we gave conditions to guarantee that all orbits in Int $\mathbb{R}_{+}^{m}$ are attracted by the closure of the same 
invariant $(m-1)$-manifold. The above results mean that the dynamics of type-K monotone and competitive systems is also 1-codimensional. We also gave the classification of the dynamical behavior of the 3-dimensional type- $\mathrm{K}$ monotone and type-K competitive Lotka-Volterra systems.

In mathematical biology, particularly in theoretical ecology, it is always a significant and essential problem to understand the mechanism for the evolution of amounts of biological species affecting each other. The theory of cooperative, competitive, type-K monotone, and type-K competitive Kolmogorov ordinary differential equation systems is a very powerful method for studying such problems in many cases. On the other hand, it is well known that spatial heterogeneities occur at all scales of the environment and all species migrate or diffuse in the environment. It becomes important to understand dispersal within the environment. When the continuous environment is considered, reaction diffusion system models are often established to describe such phenomena. When the discrete environment is considered, the models of Kolmogorov ordinary differential equation systems with migration forms can be established to describe the migration of the species between the patches. In particular, Takeuchi [16, Takeuchi and Lu [17], and Smith [12] considered the following 2-species competitive Lotka-Volterra system with migration:

$$
\begin{aligned}
\frac{d x_{1}}{d t} & =\varepsilon\left(x_{2}-x_{1}\right)+r_{1} x_{1}\left(1-x_{1} K_{1}^{-1}-a_{1} y_{1}\right), \\
\frac{d x_{2}}{d t} & =\varepsilon\left(x_{1}-x_{2}\right)+r_{2} x_{2}\left(1-x_{2} K_{2}^{-1}-a_{2} y_{2}\right), \\
\frac{d y_{1}}{d t} & =\delta\left(y_{2}-y_{1}\right)+s_{1} y_{1}\left(1-y_{1} L_{1}^{-1}-b_{1} x_{1}\right), \\
\frac{d y_{2}}{d t} & =\delta\left(y_{1}-y_{2}\right)+s_{2} y_{2}\left(1-y_{2} L_{2}^{-1}-b_{2} x_{2}\right) .
\end{aligned}
$$

Here $x_{1}, x_{2}, y_{1}, y_{2} \geq 0, x_{1}, x_{2}$ are the amounts of the species 1 on the two distinct patches $\mathrm{A}$ and $\mathrm{B}$ and $y_{1}, y_{2}$ are the amounts of the species 2 on these two patches, and all parameters are positive numbers. They showed that such systems are type-K monotone and gave conditions for the permanence of the systems.

However, consider the more general $n$-species competitive system with migration

$$
\begin{aligned}
& \frac{d x_{i}}{d t}=h_{i}\left(y_{i}-x_{i}\right)+x_{i} f_{i}(x), \\
& \frac{d y_{i}}{d t}=-h_{i}\left(y_{i}-x_{i}\right)+y_{i} g_{i}(y),
\end{aligned}
$$

where $x=\left(x_{1}, x_{2}, \cdots, x_{n}\right), y=\left(y_{1}, y_{2}, \cdots, y_{n}\right), x_{i}, y_{i}$ are the amounts of the same species $i$ on two distinct patches, $x_{i}, y_{i} \geq 0(1 \leq i \leq n)$; for any $1 \leq i \leq n, h_{i}: \mathbb{R} \rightarrow \mathbb{R}$ is a $C^{1}$ function with $h_{i}(0)=0$ and $h^{\prime}(z)>0, z \in \mathbb{R}$; for any $1 \leq i, j \leq n, i \neq j$, $\frac{\partial f_{i}}{\partial x_{j}}, \frac{\partial g_{i}}{\partial y_{j}} \leq 0$. Smith [12] pointed out: "Little is known about the case of more than two competitors. The resulting system is no longer monotone in this case." In this paper, we study the dynamical behavior of (1.4) with more than two species. It is easy to see that such a system is type-K competitive when we let $m=2 n, k=n$ in (1.2). Hence the flow generated by (1.4) is backward type-K monotone. Moreover, we can prove that almost all conclusions for type-K competitive Kolmogorov systems also hold for $n$ species competitive systems with migration; however the proofs are more difficult since the system (1.4) has the migration forms. Mainly, if the Jacobian matrix of the system is irreducible at every point in Int $\mathbb{R}_{+}^{2 n}$, then there is a defined countable family of invariant $(2 n-1)$-cells which attract all nonconvergent persistent trajectories. 
In the second part of this paper, we focus on the 2-species competitive system with migration which is a 4-dimensional system. It is well known that for the planar ordinary differential equation systems the Poincaré-Bendixson theorem holds; that is, any limit set without equilibrium is one periodic orbit. However when the dimension of the phase space is more than 2, the dynamics of the ODE system is very complicated. We can only prove the Poincaré-Bendixson theorem for a few classes of systems such as 3-dimensional monotone or competitive systems (see Smith [12]). It is interesting that we show that 2 -species competitive systems with migration are not only type-K monotone but also type-K competitive in the second part of this paper. Since the dynamics of both type-K monotone and type-K competitive systems is essentially 1-codimensional, the dynamics of 2 -species competitive systems with migration is 2-dimensional. Moreover, we prove that the Poincaré-Bendixson theorem holds for 2-species competitive systems with migration.

2. $n$-species competitive systems with migration. In this section, we consider the $n$-species competitive system with migration, which is modelled by the following group of $2 n$ ordinary differential equations:

$$
\begin{aligned}
\frac{d x_{i}}{d t} & =h_{i}\left(y_{i}-x_{i}\right)+x_{i} f_{i}(x), \\
\frac{d y_{i}}{d t} & =-h_{i}\left(y_{i}-x_{i}\right)+y_{i} g_{i}(y) .
\end{aligned}
$$

Here, $x=\left(x_{1}, x_{2}, \cdots, x_{n}\right), y=\left(y_{1}, y_{2}, \cdots, y_{n}\right) ; x_{i}, y_{i} \geq 0(1 \leq i \leq n)$; for any $1 \leq i \leq n$, $h_{i}: \mathbb{R} \rightarrow \mathbb{R}$ is a $C^{1}$ function with $h_{i}(0)=0$ and $h^{\prime}(z)>0, z \in \mathbb{R}$; for any $1 \leq i, j \leq n, i \neq j$ and any $x, y$ with $x_{i}, y_{i} \geq 0, i=1, \cdots, n, \frac{\partial f_{i}}{\partial x_{j}}, \frac{\partial g_{i}}{\partial y_{j}} \leq 0$. In the following paragraphs, we will give the definition of the general type-K competitive flow and show that the flow $\psi$ generated by (2.1) is just type-K competitive.

Let $\mathbb{R}_{+}^{m}=\left\{x \in \mathbb{R}^{m}: x_{i} \geq 0\right.$ for $\left.1 \leq i \leq m\right\}$ and Int $\mathbb{R}_{+}^{m}=\left\{x \in \mathbb{R}^{m}: x_{i}>0\right.$ for $1 \leq i \leq m\}$. For an integer $k$ with $1 \leq k \leq m$ define the set $K=\left\{x \in \mathbb{R}^{m}: x_{i} \geq 0\right.$ for $1 \leq i \leq k$ and $x_{j} \leq 0$ for $\left.k+1 \leq j \leq m\right\}$. Let Int $K$ be the interior of $K$ in $\mathbb{R}^{m}$. We know that both $\mathbb{R}_{+}^{m}$ and $K$ are cones in $\mathbb{R}^{m}$. For any two points $x, y \in \mathbb{R}_{+}^{m}$, we write $x \leq_{K} y$ and $y \geq_{K} x$ whenever $y-x \in K, x<_{K} y, y>_{K} x$ whenever $x \leq_{K} y$ and $x \neq y$, and $x \ll_{K} y$ and $y \gg_{K} x$ whenever $y-x \in \operatorname{Int} K$. For two sets $S_{1}, S_{2} \subset \mathbb{R}_{+}^{m}, S_{1} \leq_{K} S_{2}$ provided that for any $z_{1} \in S_{1}, z_{2} \in S_{2}, z_{1} \leq_{K} z_{2}$. Here $S_{1}<_{K} S_{2}, S_{1} \ll_{K} S_{2}$ can be similarly defined. Define $[x,+\infty)_{K}=\left\{y \in \mathbb{R}_{+}^{m}: y \geq_{K} x\right\}$ and $(-\infty, x]_{K}=\left\{y \in \mathbb{R}_{+}^{m}\right.$ : $\left.y \leq_{K} x\right\}$. If $x, y \in \mathbb{R}_{+}^{m}$ and $x \leq_{K}\left(\ll_{K}\right) y$, define $[x, y]_{K}=\left\{z \in \mathbb{R}_{+}^{m}: x \leq_{K} z \leq_{K} y\right\}$, $\left((x, y)_{K}=\left\{z \in \mathbb{R}_{+}^{m}: x \ll_{K} z \ll_{K} y\right\}\right)$. Similarly, we can define the notation $(x,+\infty)_{K}$ and $(-\infty, x)_{K}$.

Define $M=\{1,2, \ldots, m\}$. Let $L$ be a subset of $M$, and let $\bar{L}=M \backslash L$ be its complement in $M$. We define the set $H_{L}^{+}=\left\{x \in \mathbb{R}_{+}^{m}: x_{p}=0\right.$ for $\left.p \in \bar{L}\right\}$ and the set Int $H_{L}^{+}=\left\{x \in H_{L}^{+}: x_{p}>0\right.$ for $\left.p \in L\right\}$.

An $m \times m$ matrix $A$ is called a monotone matrix provided all off-diagonal elements of $A$ are nonnegative, and it is called a type- $K$ monotone matrix provided $A$ has the form

$$
\left(\begin{array}{rr}
A_{1} & -A_{2} \\
-A_{3} & A_{4}
\end{array}\right),
$$

in which $A_{1}$ is a $k \times k$ monotone matrix, $A_{2}$ is a $k \times(m-k)$ positive matrix, $A_{3}$ is an $(m-k) \times k$ positive matrix, and $A_{4}$ is an $(m-k) \times(m-k)$ monotone matrix. 
Here $A$ is said to be competitive (type-K competitive) provided $-A$ is monotone (type-K monotone).

Let $\psi$ be a (local) flow on $\mathbb{R}_{+}^{m}$. For any $x \in \mathbb{R}_{+}^{m}$ denote by $I_{x}$ the interval in which $\psi_{t}(x)$ exists and belongs to $\mathbb{R}_{+}^{m}$ and let $T_{x}=\inf I_{x}$; that is, for any $t \in I_{x}, \psi_{t}(x)$ exists and belongs to $\mathbb{R}_{+}^{m}$. Here $\psi$ is said to be type-K competitive or backward type$K$ monotone provided for any $x, y \in \mathbb{R}_{+}^{m}$ with $x \leq_{K} y, \psi_{t}(x) \leq_{K} \psi_{t}(y)$ for any $t$ with $\max \left(T_{x}, T_{y}\right)<t<0$, and it is said to be strongly type-K competitive on some open subset $\Omega \subset \mathbb{R}_{+}^{m}$ provided for any $x, y \in \Omega$ with $x<_{K} y$ and any $t<0$ with $\psi_{t}(x), \psi_{t}(y) \in \Omega$, $\psi_{t}(x) \ll_{K} \psi_{t}(y)$. Consider the $m$-dimensional system $\dot{x}=f(x)$ on $\mathbb{R}_{+}^{m}$. By the Kamke Theorem (see [5]), if $D f(x)$ is type-K competitive at any $x \in \mathbb{R}_{+}^{m}$, then the flow generated by this system is also type-K competitive. Furthermore if $D f(x)$ is irreducible in some open subset $\Omega \subset \mathbb{R}_{+}^{m}$, then the flow is strongly type-K competitive on $\Omega$. Obviously, the (strongly) forward type- $K$ monotone flow can be defined similarly.

Hence when we consider the cone $K=\left\{(x, y) \in \mathbb{R}^{2 n}: x_{i} \geq 0, y_{i} \leq 0\right\}$, the flow of the system (2.1) is type-K competitive in $\mathbb{R}_{+}^{2 n}$. Furthermore, in this paper we suppose that the system (2.1) is dissipative and $\Gamma$ is the attractor of the system and the Jacobian matrix of the vector field is irreducible in Int $\mathbb{R}_{+}^{2 n}$. Thus by the Kamke Theorem we can easily obtain that the system is strongly type-K competitive in Int $\mathbb{R}_{+}^{2 n}$.

Now, we define some sets which play important roles in this paper. We say that a point $z$ is in the lower boundary $\partial_{-} S$ of a set $S \subset \mathbb{R}^{2 n}$ provided there is some sequence $\left\{z_{i}\right\}$ in $S$ converging to $z$ with $z \ll_{K} z_{i}$ but no sequence $\left\{z_{i}\right\}$ in $S$ converging to $z$ with $z_{i} \ll_{K} z$, and we can define the upper boundary $\partial_{+} S$ analogously. Denote by $\mathcal{E}$ the equilibria set of (2.1). For each equilibrium $p \in \mathcal{E}$, we define the following sets in $\mathbb{R}_{+}^{2 n}$ :

$$
\begin{gathered}
R(p)=\text { the basin of repulsion of } p=\left\{z \in \mathbb{R}_{+}^{2 n}: \lim _{t \rightarrow-\infty} \psi_{t}(x)=p\right\}, \\
R_{-}(p)=\text { the basin of lower repulsion }=\left\{z \in R(p): \psi_{t}(x) \ll_{K} p \text { for some } t \in \mathbb{R}\right\}, \\
R_{+}(p)=\text { the basin of upper repulsion }=\left\{z \in R(p): \psi_{t}(x) \gg_{K} p \text { for some } t \in \mathbb{R}\right\}, \\
V_{-}(p)=\text { the lower repulsion boundary }=\partial_{-} R_{-}(p), \\
V_{+}(p)=\text { the upper repulsion boundary }=\partial_{+} R_{+}(p) .
\end{gathered}
$$

Notice that we have defined the same sets in our paper [9] for type-K competitive Kolmogorov systems. In fact, in this paper we will prove that $n$-species competitive systems with migration, particularly the persistent trajectories of the system, have almost the same properties as those of type-K competitive Kolmogorov systems. In the rest of this section, we will present these properties and leave all the proofs for the appendix.

First, we have

Theorem 2.1. Consider the $n$-species competitive system with migration. Let $p, q \in \mathcal{E}$. Then we have the following.

(a) $R_{-}(p), V_{-}(p)$ are invariant; moreover, they are bounded subsets of Int $\mathbb{R}^{2 n}$.

(b) $R_{-}(p)$ is order-convex with respect to the order $\leq_{K}$ and open in $\mathbb{R}_{+}^{2 n}$.

(c) $R_{-}(p) \cap R_{-}(q)$ and $V_{-}(p) \cap V_{-}(q)$ are empty if $p \neq q$.

(d) $V_{-}(p)$ is unordered with respect to $<_{K}$. 
(e) Let $P_{E}: \mathbb{R}^{2 n} \rightarrow E$ be an orthogonal projection onto a hyperplane $E$ orthogonal to a vector $v \gg_{K} 0$. Then $\left.P_{E}\right|_{V_{-}(p)}$ is a homeomorphism $g_{E}: V_{-}(p) \rightarrow U$ onto an open subset $U \subset E ; g_{E}$ and $\left(g_{E}\right)^{-1}$ are Lipschitz.

(f) If $R_{-}(p)$ is nonempty, then $R_{-}(p)$ is an open $2 n$-cell and $V_{-}(p)$ is an open $(2 n-1)$ cell.

(g) If $z \in \operatorname{clos} V_{-}(p)$ and $z<_{K} p$, then $z \in V_{-}(p)$, where $\operatorname{clos} V_{-}(p)$ means the closure of $V_{-}(p)$ in $\mathbb{R}_{+}^{2 n}$.

(h) Analogous results hold for $R_{+}(p)$ and $V_{+}(p)$.

Here, $S$ is an open $m$-cell means that $S$ is homeomorphic to $\mathbb{R}^{m}$.

REMARK 2.2. In Theorem 2.1, we prove the (total) invariance of the sets $R_{-}(p), R_{+}(p)$, $V_{-}(p)$, and $V_{+}(p)$ in a strong sense; that is, a set $\Omega$ is invariant means that if $z \in \Omega$, then for any $t \in(-\infty,+\infty), \psi_{t}(z)$ exists and belongs to $\Omega$. However since the system is dissipative and only the set $\mathbb{R}_{+}^{2 n}$ is positively invariant, an orbit not belonging to $\Gamma$ may not extend to $t=-\infty$. We use invariance in a weak sense in the rest of this paper; that is, a set $\Omega$ is invariant means that if $z \in \Omega$, then for any $t \in\left(T_{z},+\infty\right), \psi_{t}(z) \in \Omega$.

To understand more fully the dynamics of the system (2.1), we still need to consider the case of " $\infty$ ". Moreover, since $\pm \infty$ with respect to $\leq_{K}$ in $\mathbb{R}_{+}^{2 n}$ are different, we need to define the basin of lower (upper) repulsion of $\infty$ and its lower (upper) boundary.

$$
\begin{aligned}
& R(\infty)=\mathbb{R}_{+}^{2 n} \backslash \Gamma, \\
& \tilde{R}_{-}(\infty)=\left\{z \in R(\infty) \cap \operatorname{Int} \mathbb{R}_{+}^{2 n}: \text { either } T_{z}>-\infty,\left(\psi_{T_{z}}(z)\right)_{k}>0, k=1, \cdots, n,\right. \\
& \text { and }\left(\psi_{T_{z}}(z)\right)_{i}=0 \text { for some } n+1 \leq i \leq 2 n \text {, or } \sup _{t \in\left(T_{z}, 0\right)}\left(\psi_{t}(z)\right)_{k}<+\infty \text {, } \\
& \left.k=n+1, \cdots, 2 n \text { and } \sup _{t \in\left(T_{z}, 0\right)}\left(\psi_{t}(z)\right)_{i}=+\infty \text { for some } 1 \leq i \leq n\right\}, \\
& \tilde{R}_{+}(\infty)=\left\{z \in R(\infty) \cap \operatorname{Int} \mathbb{R}_{+}^{2 n}: \text { either } T_{z}>-\infty,\left(\psi_{T_{z}}(z)\right)_{k}>0, k=n+1, \cdots, 2 n,\right. \\
& \text { and }\left(\psi_{T_{z}}(z)\right)_{i}=0 \text { for some } 1 \leq i \leq n \text {, or } \sup _{t \in\left(T_{z}, 0\right)}\left(\psi_{t}(z)\right)_{k}<+\infty \text {, } \\
& \left.k=1, \cdots, n \text { and } \sup _{t \in\left(T_{z}, 0\right)}\left(\psi_{t}(z)\right)_{i}=+\infty \text { for some } n+1 \leq i \leq 2 n\right\}, \\
& R_{-}(\infty)=\text { the interior of } \tilde{R}_{-}(\infty) \text { in } \mathbb{R}_{+}^{2 n} \text {, } \\
& R_{+}(\infty)=\text { the interior of } \tilde{R}_{+}(\infty) \text { in } \mathbb{R}_{+}^{2 n} \text {, } \\
& V_{-}(\infty)=\operatorname{Int} \mathbb{R}_{+}^{2 n} \cap \partial_{-} R_{-}(\infty), \\
& V_{+}(\infty)=\operatorname{Int} \mathbb{R}_{+}^{2 n} \cap \partial_{+} R_{+}(\infty) .
\end{aligned}
$$

Proposition 2.3. $\tilde{R}_{-}(\infty)$ and $\tilde{R}_{+}(\infty)$ are nonempty and ordered convex. If $z=(x, y) \in$ $\tilde{R}_{-}(\infty)$, then for any $t \in\left(T_{z},+\infty\right), \psi_{t}(z) \in \tilde{R}_{-}(\infty)$. Moreover, $z=(x, y) \in \tilde{R}_{-}(\infty)$ implies $[z,+\infty)_{K} \cap \operatorname{Int} \mathbb{R}_{+}^{2 n} \subset \tilde{R}_{-}(\infty) ; z=(x, y) \in \tilde{R}_{+}(\infty)$ implies $(-\infty, z]_{K} \cap \operatorname{Int} \mathbb{R}_{+}^{2 n} \subset$ $\tilde{R}_{+}(\infty)$. Moreover, $R_{-}(\infty)$ also has the above properties and is open in $\operatorname{Int} \mathbb{R}_{+}^{2 n}$. Here $V_{-}(\infty)$ is invariant and unordered by $<_{K}$. For $\tilde{R}_{+}(\infty), R_{+}(\infty), V_{+}(\infty)$, the analogous conclusions hold. 
Proposition 2.4. $R_{-}(\infty)$ and $R_{+}(\infty)$ are homeomorphic to $\mathbb{R}^{2 n} ; V_{-}(\infty)$ and $V_{+}(\infty)$ are homeomorphic to $\mathbb{R}^{2 n-1}$.

To know more about the properties of the limit sets, we will extend the limit set dichotomy of the strongly monotone system (see 2]) to the competitive system with migration.

Proposition 2.5 ( $\alpha$-limit set dichotomy of the competitive system with migration). Suppose that $z, z^{\prime} \in \mathbb{R}_{+}^{2 n}$ with $z<_{K} z^{\prime}$, and suppose that $\alpha(z), \alpha\left(z^{\prime}\right)$ exist and at least one of them is contained in Int $\mathbb{R}_{+}^{2 n}$. Then either $\alpha(z) \ll_{K} \alpha\left(z^{\prime}\right)$ or $\alpha(z)=\alpha\left(z^{\prime}\right) \subset \mathcal{E}$. Moreover, both $\alpha(z)$ and $\alpha\left(z^{\prime}\right)$ are contained in $\operatorname{Int} \mathbb{R}_{+}^{2 n}$.

Based on Proposition 2.5. we can obtain the following theorem about the limit sets of the persistent trajectories.

Theorem 2.6. Suppose that $\mathcal{K} \subset \operatorname{Int} \mathbb{R}_{+}^{2 n}$ is an $\alpha$ - or $\omega$-limit set which is not a singleton. Then there are $p, q \in \mathcal{E} \cup\{\infty\}$ such that

$$
\mathcal{K} \subset V_{-}(q), \mathcal{K} \subset V_{+}(p) .
$$

REmark 2.7. In Theorem 2.6. $V_{-}(q)$ is nonempty and so is $R_{-}(q)$. For each $q$ with nonempty $R_{-}(q)$, there is a set $(a, q)_{K}$ such that there is no equilibrium in $(a, q)_{K}$. This implies that the number of the equilibria with the nonempty basin of lower repulsion is countable.

Theorem 2.8. Suppose that $\omega(z) \subset V_{-}(q)$ for some $q \in \mathcal{E} \cup\{\infty\}$. Then there is some $z^{\prime} \in V_{-}(q)$ such that $\left\|\psi_{t}\left(z^{\prime}\right)-\psi_{t}(z)\right\| \rightarrow 0$ as $t \rightarrow \infty$.

Corollary 2.9. Suppose that $\frac{\partial f_{i}}{\partial x_{i}}<0$ and $\frac{\partial g_{i}}{\partial y_{i}}<0$ hold for any $z \in \mathbb{R}_{+}^{2 n}$ and $i=$ $1,2, \cdots, n$. Then for any limit set $\mathcal{K} \subset \operatorname{Int} \mathbb{R}_{+}^{2 n}$, we have

$$
\mathcal{K} \subset V_{-}(\infty), \mathcal{K} \subset V_{+}(\infty) .
$$

Furthermore, if $\mathcal{K}=\omega(z)$, then there are some $z^{\prime} \in V_{-}(\infty)$ with $\left\|\psi_{t}\left(z^{\prime}\right)-\psi_{t}(z)\right\| \rightarrow 0$ and some $z^{\prime \prime} \in V_{+}(\infty)$ with $\left\|\psi_{t}\left(z^{\prime \prime}\right)-\psi_{t}(z)\right\| \rightarrow 0$ as $t \rightarrow \infty$.

3. 2-Species competitive systems with migration. In this section, we consider 2 -species competitive systems with migration. We will prove that the dynamical behavior of such a system is 2-codimensional and therefore the Poincaré-Bendixson theorem holds for this system.

Rewrite the 2-species competitive systems with migration in the following form:

$$
\begin{aligned}
\frac{d x_{1}}{d t} & =\varepsilon\left(x_{2}-x_{1}\right)+r_{1} x_{1}\left(1-x_{1} K_{1}^{-1}-a_{1} y_{1}\right), \\
\frac{d x_{2}}{d t} & =\varepsilon\left(x_{1}-x_{2}\right)+r_{2} x_{2}\left(1-x_{2} K_{2}^{-1}-a_{2} y_{2}\right), \\
\frac{d y_{1}}{d t} & =\delta\left(y_{2}-y_{1}\right)+s_{1} y_{1}\left(1-y_{1} L_{1}^{-1}-b_{1} x_{1}\right), \\
\frac{d y_{2}}{d t} & =\delta\left(y_{1}-y_{2}\right)+s_{2} y_{2}\left(1-y_{2} L_{2}^{-1}-b_{2} x_{2}\right) .
\end{aligned}
$$

Here $x_{1}, x_{2}, y_{1}, y_{2} \geq 0, x_{1}, x_{2}$ are the amounts of the species 1 on the two isolated domains $\mathrm{A}$ and $\mathrm{B}$ and $y_{1}, y_{2}$ are the amounts of the species 2 on $\mathrm{A}$ and $\mathrm{B}$, and all parameters are positive numbers. Notice that the sense of the symbols $x_{1}, x_{2}, y_{1}, y_{2}$ is a little different from those in the previous section. 
Smith [12] shows that when we consider the type-K cone $K_{1}=\left\{\left(x_{1}, x_{2}, y_{1}, y_{2}\right) \in \mathbb{R}^{4}\right.$ : $\left.x_{1}, x_{2} \geq 0, y_{1}, y_{2} \leq 0\right\}$, the system is type-K monotone. He also gives the conditions for the permanence of the system. It is interesting that we find that when we consider the cone $K_{2}=\left\{\left(x_{1}, x_{2}, y_{1}, y_{2}\right) \in \mathbb{R}^{4}: x_{1}, y_{1} \geq 0, x_{2}, y_{2} \leq 0\right\}$, the system is also type-K competitive. Since the dynamics of both the type-K monotone systems and the type-K competitive systems is 1-codimensional, the dynamics of 2-species competitive systems with migration is 2-dimensional. Precisely, we have

Theorem 3.1. The flow on a compact limit set of the system (3.1) in Int $\mathbb{R}_{+}^{4}$ is topologically equivalent to a flow on a compact invariant set of a Lipschitz system of differential equations in $\mathbb{R}^{2}$.

Proof. We only consider the $\omega$-limit set. The $\alpha$-limit set is similar. We use $\mathcal{K}$ to denote the $\omega$-limit set of $z$. Because the system is type-K monotone with respect to $\leq_{K_{1}}$ and type-K competitive with respect to $\leq_{K_{2}}$ and the Jacobian matrix of the vector field is irreducible in Int $\mathbb{R}_{+}^{4}$, we know that $\mathcal{K}$ is an unordered set by both $<_{K_{1}}$ and $<_{K_{2}}$.

Let

$$
v_{1}=\left(\frac{1}{2}, \frac{1}{2},-\frac{1}{2},-\frac{1}{2}\right), \quad \text { and } \quad v_{2}=\left(\frac{1}{2},-\frac{1}{2}, \frac{1}{2},-\frac{1}{2}\right) .
$$

It is easy to see that the subspace $\left\{a v_{1}+b v_{2}=\left(\frac{a+b}{2}, \frac{a-b}{2}, \frac{b-a}{2},-\frac{a+b}{2}\right), a, b \in \mathbb{R}\right\}$ is included in $\pm K_{1} \cup \pm K_{2}$. Let $H$ be the 2-dimensional plane orthogonal to $v_{1}, v_{2}$, and let $\mathcal{Q}$ be the orthogonal projection from $\mathbb{R}^{4}$ to $H$; that is, $\mathcal{Q} z=z-\left(z \cdot v_{1}\right) v_{1}-\left(z \cdot v_{2}\right) v_{2}$. We claim that the restriction of $\mathcal{Q}$ to $\mathcal{K}, Q=\left.\mathcal{Q}\right|_{\mathcal{K}}$, is one to one. Otherwise, there are $z, z^{\prime} \in \mathcal{K}$ such that $Q\left(z-z^{\prime}\right)=0$; that is,

$$
z-z^{\prime}=\left(z \cdot v_{1}\right) v_{1}+\left(z \cdot v_{2}\right) v_{2}-\left(z^{\prime} \cdot v_{1}\right) v_{1}-\left(z^{\prime} \cdot v_{2}\right) v_{2} .
$$

Simply denote $z-z^{\prime}=a v_{1}+b v_{2}$ where at least one of $a, b$ is not zero. This contradicts that $\mathcal{K}$ is unordered. In what follows, we want to prove that $Q$ is a homeomorphism. We only need to prove that $Q^{-1}$ is continuous. In fact, we will prove that $Q^{-1}$ is Lipschitz continuous. Suppose not. Then there are two sequences $\left\{z_{n}\right\},\left\{z_{n}^{\prime}\right\} \subset \mathcal{K}, z_{n} \neq z_{n}^{\prime}$, for $n=1,2, \cdots$, such that $\frac{\left|Q\left(z_{n}\right)-Q\left(z_{n}^{\prime}\right)\right|}{\left|z_{n}-z_{n}^{\prime}\right|} \rightarrow 0$. Equivalently,

$$
\left|\left(z_{n}-z_{n}^{\prime}\right)-\left(v_{1} \cdot\left(z_{n}-z_{n}^{\prime}\right)\right) v_{1}-\left(v_{2} \cdot\left(z_{n}-z_{n}^{\prime}\right)\right) v_{2}\right| /\left|z_{n}-z_{n}^{\prime}\right| \rightarrow 0 .
$$

Let $w_{n}=\left(z_{n}-z_{n}^{\prime}\right) /\left|z_{n}-z_{n}^{\prime}\right|$. Then $\left|w_{n}-\left(v_{1} \cdot w_{n}\right) v_{1}-\left(v_{2} \cdot w_{n}\right) v_{2}\right| \rightarrow 0$. Passing to subsequences, we suppose that $w_{n} \rightarrow w, z_{n} \rightarrow z, z_{n}^{\prime} \rightarrow z^{\prime}$. If $z \neq z^{\prime}$, we have $\left(z-z^{\prime}\right) /$ $\left|z-z^{\prime}\right|=w=\left(v_{1} \cdot w\right) v_{1}+\left(v_{2} \cdot w\right) v_{2}$ and $|w|=1$. This contradicts that $\mathcal{K}$ is unordered. Consider the case where $z=z^{\prime}$ and suppose that $w \in K_{1}$ without loss of generality. Denote by $\psi$ the flow generated by the system and by $\psi_{1}$ the time- 1 mapping. Because $D_{z} \psi_{1}(z)$ is a strongly positive linear mapping with respect to $K_{1}, D_{z} \psi_{1}(z) w \in \operatorname{Int} K_{1}$. Moreover, by the smoothness of the vector field, there is some $\epsilon$ such that for any points $z^{\prime \prime}, w^{\prime \prime}$ if $\left|z-z^{\prime \prime}\right| \leq \epsilon$ and $\left|w-w^{\prime \prime}\right| \leq \epsilon$, then $D_{z} \psi_{1}\left(z^{\prime \prime}\right) w^{\prime \prime} \in \operatorname{Int} K_{1}$. Since $w_{n}=$ $\left(z_{n}-z_{n}^{\prime}\right) /\left|z_{n}-z_{n}^{\prime}\right| \rightarrow w$ and $z_{n} \rightarrow z, z_{n}^{\prime} \rightarrow z^{\prime}$, there is some $n$ such that $\left|w_{n}-w\right| \leq \epsilon$ and

$$
\psi_{1}\left(z_{n}\right)-\psi_{1}\left(z_{n}^{\prime}\right)=\left|z_{n}-z_{n}^{\prime}\right| \int_{0}^{1} D_{z} \psi_{1}\left(\tau z_{n}+(1-\tau) z_{n}^{\prime}\right) d \tau \cdot w_{n} \gg_{K_{1}} 0,
$$


contradicting that $\mathcal{K}$ is unordered with respect to $<_{K_{1}}$. For the case where $w \in K_{2}$, we only need to consider the time- $(-1)$ mapping $\psi_{-1}$. A similar conclusion holds.

Hence, we have proved that $Q$ is a Lipschitz homeomorphism between $\mathcal{K}$ and $Q(\mathcal{K})$. For any $\bar{z} \in Q(\mathcal{K})$, there is a unique $z \in \mathcal{K}$ such that $\bar{z}=Q(z)$. Let $F$ be the vector field of the system (3.1), $G(\bar{z})=Q\left(F\left(Q^{-1}(\bar{z})\right)\right)$ be the vector field on $Q(\mathcal{K})$, and let $\psi$ be the flow generated by the system (3.1). Then $\theta_{t}(\bar{z})=Q\left(\psi_{t}(z)\right), z \in \mathcal{K}$, is the flow generated by the vector field $G$. Moreover, $G$ can be extended to a Lipschitz vector field on $H$ since $G$ is Lipschitz.

Theorem 3.2. A compact limit set of the system (3.1) that contains no equilibrium is one periodic orbit.

Proof. We still use $\mathcal{K}$ to denote the $\omega$-limit set of $z$. We only need to consider the case that $z \in \operatorname{Int} \mathbb{R}_{+}^{4}$ and $\mathcal{K} \subset \operatorname{Int} \mathbb{R}_{+}^{4}$ since if $\mathcal{K} \not \subset$ Int $\mathbb{R}_{+}^{4}$, then $\mathcal{K}$ contains an equilibrium. By Theorem 3.1 the flow $\psi$ generated by the system (3.1) on $\mathcal{K}$ is topologically equivalent to the flow $\theta$ on $Q(\mathcal{K})$. Because $\mathcal{K}$ contains no equilibria, neither does $Q(\mathcal{K})$. The PoincaréBendixson theorem implies that $Q(\mathcal{K})$ consists of periodic orbits and, possibly, entire orbits whose $\omega$ - and $\alpha$-limit sets are periodic orbits in $Q(\mathcal{K})$. Furthermore, $Q(\mathcal{K})$ consists entirely of periodic orbits by chain recurrence of $Q(\mathcal{K})$ with respect to the flow $\theta$ (see Smith [12]).

Finally, we prove that $Q(\mathcal{K})$ only consists of a periodic orbit. Otherwise $Q(\mathcal{K})$ consists of an annulus of uncountably many periodic orbits. Let the periodic orbit $C \subset \mathcal{K}$, and let $Q(C)$ be contained in the interior of $Q(\mathcal{K})$ in $\mathbb{R}^{2}$. Fix $a, b \in \mathcal{K} \backslash C$ such that $Q(a), Q(b)$ belong to different components of $Q(\mathcal{K}) \backslash Q(C)$. It is easy to see that $\mathcal{Q}\left(\psi_{t}(z)\right)$ must cross $Q(C)$ at a sequence of times $t_{k} \rightarrow+\infty$. Therefore, there is a sequence $\left\{z_{k}\right\}_{1}^{+\infty} \subset C$ such that

(1) $z_{k}<_{K_{1}} \psi_{t_{k}}(z)$ or

(2) $z_{k}>_{K_{1}} \psi_{t_{k}}(z)$ or

(3) $z_{k}<_{K_{2}} \psi_{t_{k}}(z)$ or

(4) $z_{k}>_{K_{2}} \psi_{t_{k}}(z)$.

Passing to a subsequence, we can suppose that (1) always holds or (2) always holds or (3) always holds or (4) always holds for all $k$. When (1) always holds, we can assume $z_{k} \ll_{K_{1}} \psi_{t_{k}}(z)$ since $z \in \operatorname{Int} \mathbb{R}_{+}^{4}$. Then $z_{k} \in \mathcal{K}$ implies that there is some sufficiently large number $t$ such that $\psi_{t}(z)$ and $z_{k}$ are sufficiently close, and hence $\psi_{t}(z) \ll_{K_{1}} \psi_{t_{k}}(z)$ and moreover, $\mathcal{K}$ is an equilibrium, a contradiction. Hence (1) cannot hold for any sufficiently large integer $k$. Similarly, (2) cannot hold. When (3) always holds, since $z \in \operatorname{Int} \mathbb{R}_{+}^{4}$, we can assume $z_{k} \ll_{K_{2}} \psi_{t_{k}}(z)$. Since the system is backward type-K monotone under the order $K_{2}$, for any $t>0$ there is $t_{k}>t$ such that $w=\psi_{t-t_{k}}\left(z_{k}\right)<_{K} \psi_{t-t_{k}}\left(\psi_{t_{k}}(z)\right)=\psi_{t}(z)$. This means that any point in the $\omega$-limit set $\mathcal{K}$ is larger than one point in $C$ by $<_{K_{2}}$. Similarly, if (4) always holds, then any point in $\mathcal{K}$ is less than one point in $C$ by $<_{K_{2}}$. Choose the periodic orbits $C_{1}, C_{2}, C_{3}$ as we chose $C$ above. Then any point in $\mathcal{K}$ and the points in two of $C_{1}, C_{2}, C_{3}$ (suppose $C_{1}, C_{2}$ ) have the same order relation by $<_{K_{2}}$. Let $y_{1} \in C_{1} \subset \mathcal{K}$. Then there is some $y_{2} \in C_{2}$ such that $y_{1}<_{K_{2}} y_{2}$. Similarly, there is some $y_{3} \in C_{1}$ such that $y_{2}<_{K_{2}} y_{3}$. Hence $y_{1}<_{K_{2}} y_{3}$. It contradicts that the periodic orbit $C_{1}$ is unordered by $<_{K_{2}}$. 


\section{Appendix.}

Proof of Theorem 2.1. (a) First, we claim that $R_{-}(p) \neq \emptyset$ implies $p \in \operatorname{Int} \mathbb{R}_{+}^{2 n}$. In fact, suppose that $p=\left(p_{1}, \cdots, p_{n}, p_{n+1}, \cdots, p_{2 n}\right)$. For the sake of contradiction, let $p_{i}=0,1 \leq i \leq n$. The case $n+1 \leq i \leq 2 n$ is similar. If $p_{i+n}>0$, then for any $t>0$, the $i$ th element of $\psi_{t}(p)$ is larger than 0 by the form of the equations. So $p$ cannot be an equilibrium. Otherwise, if $p_{i+n}=0$, then we cannot find any point $z \in \mathbb{R}^{2 n}$ with $z \ll_{K} p$ or $z \gg_{K} p$. Hence $R_{-}(p)$ is empty. This shows our claim.

Next, suppose that $z \in R_{-}(p)$. It is easy to see that for any $t<0$ with $|t|$ sufficiently large $\psi_{t}(z) \in \operatorname{Int} \mathbb{R}_{+}^{2 n}$ since $p \in \operatorname{Int} \mathbb{R}_{+}^{2 n}$. This means that $T_{z}=-\infty$. Hence, $R_{-}(p)$ is invariant by the definition and the backward type-K monotonicity. Moreover, $R_{-}(p) \subset$ Int $\mathbb{R}_{+}^{2 n}$.

Consider $z \in V_{-}(p)$. Let $z_{k} \in R_{-}(p), z_{k} \rightarrow z$ as $k \rightarrow+\infty$. We know $T_{z_{k}}=-\infty$, so $T_{z}=-\infty$ also. By the definition and the strong backward monotonicity there is some $t \in \mathbb{R}$ such that $\psi_{t}(z) \ll_{K} p$. Without loss of generality, assume $z \ll_{K} p$. Then $(z, p)_{K} \subset R_{-}(p)$. For any $t>0$ to prove $\psi_{t}(z) \in V_{-}(p)$, suppose that $z_{k} \rightarrow \psi_{t}(z)$ as $k \rightarrow+\infty$ and $z_{k} \gg_{K} \psi_{t}(z)$. Then $\psi_{-t}\left(z_{k}\right) \gg_{K} z$ and $\psi_{-t}\left(z_{k}\right) \rightarrow z$. This means that $\psi_{-t}\left(z_{k}\right) \in(z, p)_{K} \subset R_{-}(p)$ and hence $z_{k} \in R_{-}(p)$ for any sufficiently large integer $k$ since $R_{-}(p)$ is invariant. Furthermore, we show that there is no sequence $\left\{z_{k}\right\}_{k=1}^{\infty} \subset$ $R_{-}(p), z_{k} \rightarrow \psi_{t}(z)$ as $k \rightarrow+\infty$ and $z_{k} \ll_{K} \psi_{t}(z)$. Otherwise, the strong backward monotonicity implies $\psi_{-t}\left(z_{k}\right) \ll_{K} z$ and $\psi_{-t}\left(z_{k}\right) \rightarrow z$. But $z \in V_{-}(p)$, a contradiction. Hence, $V_{-}(p)$ is invariant.

Moreover, suppose that $z=\left(z_{1}, \cdots, z_{n}, z_{n+1}, \cdots, z_{2 n}\right)$. We claim that $z_{i}>0$ for $1 \leq i \leq 2 n$. For the sake of contradiction, let $z_{i}=0,1 \leq i \leq n$. The case $n+1 \leq i \leq 2 n$ is similar. If $z_{i+n}>0$, then for any $t<0$ with $|t|$ sufficiently small, the $i$ th element of $\psi_{t}(z)$ is smaller than 0 by the form of the equations, contradicting the invariance of $V_{-}(p)$. Otherwise, if $z_{i+n}=0$, then we cannot find any point $z^{\prime} \in \mathbb{R}_{+}^{2 n}$ with $z^{\prime} \gg_{K} p$. Hence $V_{-}(p) \subset$ Int $\mathbb{R}_{+}^{2 n}$. Last, since the system is dissipative, $R_{-}(p)$ is bounded and so is $V_{-}(p)$.

(b) First, if $z \in R_{-}(p)$ and $z \ll_{K} p$, then $[z, p)_{K} \subset R_{-}(p)$ by the backward monotonicity. Suppose that $z_{1}<_{K} z_{2}<_{K} z_{3}$ and $z_{1}, z_{3} \in R_{-}(p)$. Then the backward monotonicity implies there is some $t \in \mathbb{R}$ such that $\psi_{t}\left(z_{1}\right)<_{K} \psi_{t}\left(z_{2}\right)<_{K} \psi_{t}\left(z_{3}\right) \ll_{K} p$. This means that $\psi_{t}\left(z_{2}\right) \in R_{-}(p)$ and so is $z_{2}$.

(c) It is obvious that $R_{-}(p) \cap R_{-}(q)=\emptyset$. Consider $V_{-}(p) \cap V_{-}(q)$. For the sake of contradiction, let $z \in V_{-}(p) \cap V_{-}(q)$. There are $z_{k} \in R_{-}(p), z_{k}^{\prime} \in R_{-}(q)$ such that $z_{k}, z_{k}^{\prime} \gg_{K} z$ for $k=1,2, \cdots$ and $z_{k}, z_{k}^{\prime} \rightarrow z$ as $k \rightarrow+\infty$. Hence, passing to subsequences, we can obtain $z_{k+1} \ll_{K} z_{k}^{\prime} \ll_{K} z_{k}$ for $k=1,2, \cdots$. This implies $z_{k}^{\prime} \in R_{-}(p)$ by the order convexity of $R_{-}(p)$, contradicting $R_{-}(p) \cap R_{-}(q)=\emptyset$.

(d) Suppose, for the sake of contradiction, that there are $z, z^{\prime} \in V_{-}(p)$ with $z<_{K} z^{\prime}$. Since $z, z^{\prime} \in \operatorname{Int} \mathbb{R}_{+}^{2 n}, \psi_{t}(z) \ll_{K} \psi_{t}\left(z^{\prime}\right)$ for $t<0$ by the strong backward monotonicity. Without loss of generality, assume $z \ll_{K} z^{\prime}$. There are $z_{k}, z_{k}^{\prime} \in R_{-}(p)$ such that $z_{k} \gg_{K}$ $z, z_{k}^{\prime} \gg_{K} z^{\prime}$ for $k=1,2, \cdots$ and $z_{k} \rightarrow z, z_{k}^{\prime} \rightarrow z^{\prime}$ as $k \rightarrow+\infty$. Hence it is easy to find some sufficiently large integer $k$ such that $z_{k}<z^{\prime}<z_{k}^{\prime}$ and then $z^{\prime} \in R_{-}(p)$. But $R_{-}(p)$ is open and then $R_{-}(p) \cap V_{-}(p)=\emptyset$. This is a contradiction. 
(e) Actually, Proposition 2.6 of Hirsch [3] implies (e). To complete this paper, we repeat the proof here.

To see that $g$ is injective, suppose $g(z)=g\left(z^{\prime}\right)$. Then $z=z^{\prime}+\lambda v$ for some $\lambda \in \mathbb{R}$. Since $v \gg_{K} 0$ and no two points of $V_{-}(p)$ can be related by $<_{K}$, it follows that $\lambda=0$; therefore $g$ is injective. The image of $g$ is open in $E$ and $g^{-1}$ is continuous. To see this, fix $a \in V_{-}(p)$ and set $g(a)=b \in E$. Choose $c \gg_{K} a$ so that $(a, c)_{K} \subset R_{-}(p)$. It is easy to show that $P_{E}\left((a, c)_{K}\right)$ is a neighborhood in $E$ of $b$. For any $z \in P_{E}\left((a, c)_{K}\right)$ let $L_{z}$ denote the line through $z$ parallel to $u$. Then $L_{z} \cap B_{-}(p)$ has the greatest lower bound $w \in L_{z}$ because $R_{-}(p)$ is bounded below, $v \in \operatorname{Int} K$, and $L_{z}$ meets $(a, c)_{K}$. It is not hard to see that $w \in V_{-}(p)$ and $g(w)=z$. Thus $g\left(V_{-}(p)\right)$ is open in $E$. From this construction of $w$ as $g^{-1}(z)$ it is easy to see that $g^{-1}$ is continuous. This proves $g$ is a homeomorphism onto an open set in $E$.

Since $P_{E}$ has Lipschitz constant 1 , so does $g$. We show that $g^{-1}$ has a Lipschitz constant which depends only on $v$. Denote by $S_{E}$ the set of all unit vectors in the linear subspace $E$. It is easy to see that $S_{E}$ is disjoint from $K$ and that there exists some number $\mu>0$ with the following property. If $z \in S_{E}, \lambda \in \mathbb{R}$, and $z+\lambda v \notin K$, then $|\lambda|<\mu$.

We show that $1+\mu$ is a Lipschitz constant for $g^{-1}$. Fix two points $a, b \in E$. Set $a-b=w \in E$ and $g^{-1}(a)-g^{-1}(b)=u$. Then $u=w+\rho v$ for some $\rho \in \mathbb{R}$. Notice that $u \notin K$. Consider the identity $u /|w|=w /|w|+(\rho /|w|) v$. Since $u /|w| \notin K$, we find that $\rho /|w|<\mu$. By the triangle inequality we therefore get $|u| /|w|<1+\mu$.

(f) Suppose that $R_{-}(p)$ is nonempty. Then there is some $a \in R_{-}(p)$ such that $a \ll_{K} p$; fix this $a$. By the order convexity of $R_{-}(p)$ and the strong backward monotonicity we have $[a, p] \backslash p \subset R_{-}(p) \subset \operatorname{Int} \mathbb{R}_{+}^{2 n}$. Moreover, for any $b \in R_{-}(p)$, there is some $\tau_{b}<0$ such that for any $t \in\left(-\infty, \tau_{b}\right], \psi_{t}(b) \in(a, p)_{K}$. In particular, there is some positive integer $m$ such that $\psi_{m \tau_{a}}(b) \in(a, p)_{K}$. Moreover, the backward monotonicity implies $\psi_{\tau_{a}}\left((a, p)_{K}\right) \subset$ $(a, p)_{K}$. Let $U_{m}=\psi_{-m \tau_{a}}\left((a, p)_{K}\right), m=0,1, \cdots$. Then $R_{-}(p)=\bigcup_{m=0}^{\infty} U_{m}$ and $U_{k} \subset U_{m}$ if $k \leq m$. Moreover, $U_{m}=\psi_{-m T_{a}}\left((a, p)_{K}\right)$ is an open $2 n$ cell since it is homeomorphic to $(a, p)_{K}$. A theorem of Brown (1961) implies that such a union is also an open $n$-cell (see [3]).

To prove the second statement, fix a number $\delta$ in the range $0<\delta<\min \left\{\left|p_{i}-a_{i}\right|, i=\right.$ $1,2, \cdots, 2 n\}$ and define the set

$$
S_{\delta}(p)=\left\{z \in \mathbb{R}^{2 n}, z \leq_{K} p \text { and }|z-p|=\delta\right\}
$$

where $|\cdot|$ is the Euclidean norm, and let $L_{\delta}(p)=\left\{z \in S_{\delta}(p), z \ll_{K} p\right\}$. It is easy to see that $S_{\delta}(p)$ is a closed $(2 n-1)$-cell and $L_{\delta}(p)$ is an open $(2 n-1)$-cell.

Consider for each $z \in S_{\delta}(p)$ the ray $R_{z}$ through $z$ emanating from $p$. Since $R_{-}(p)$ is order convex and bounded, $R_{z} \cap R_{-}(p)$ is a bounded open interval with one endpoint at $p$. It is easy to see that the other endpoint, denoted by $g(z)$, belongs to $V_{-}(p)$ and that the resulting map $g: S_{\delta}(p) \rightarrow V_{-}(p)$ sends $S_{\delta}(p)$ homeomorphically onto the set

$$
D(p)=\left\{z \in V_{-}(p): x<_{K} p\right\}
$$


while $g$ maps $L_{\delta}(p)$ homeomorphically onto the set

$$
D^{0}(p)=\left\{z \in D(p): x \ll_{K} p\right\} .
$$

Therefore $D^{0}(p)$ is an open $(2 n-1)$-cell.

Now observe that for any $z \in V_{-}(p)$ there is a number $t_{z}<0$ such that $\psi_{t}(z) \in D^{0}(p)$ for all $t<t_{z}$. This means $V_{-}(p)=\bigcup_{t>0} \psi_{t}\left(D^{0}(p)\right)$. Furthermore, $D^{0}(p) \subset \psi_{t}\left(D^{0}(p)\right)$ for any $t>0$. This implies $\psi_{t}\left(D^{0}(p)\right) \subset \psi_{t^{\prime}}\left(D^{0}(p)\right)$ if $t<t^{\prime}$. By Brown's theorem, $V_{-}(p)$ is an open $(2 n-1)$-cell.

(g) First, $p, z \in \operatorname{Int} \mathbb{R}_{+}^{2 n}$. Without loss of generality, by the strong backward monotonicity and invariance of $V_{-}(p)$, suppose that $z \ll_{K} p$. By the proof of (f), $z \in V_{-}(p)$.

Proof of Proposition 2.3 . We can easily see that $\tilde{R}_{-}(\infty)$ is nonempty since for any $t>0$ and any $z=\left(x_{1}, \cdots, x_{n}, 0, \cdots, 0\right)$ with $x_{i} \neq 0,1 \leq i \leq n$, we have $\psi_{t}(z) \in \tilde{R}_{-}(\infty)$. Next, we will prove that if $z \in \tilde{R}_{-}(\infty)$, then $[z,+\infty)_{K} \cap \operatorname{Int} \mathbb{R}_{+}^{2 n} \subset \tilde{R}_{-}(\infty)$. Suppose that $z^{\prime} \in[z,+\infty)_{K}$, by the backward monotonicity, $T_{z} \leq T_{z^{\prime}}$, and for any $t \in\left[T_{z^{\prime}}, 0\right)$, $\psi_{t}(z) \leq_{K} \psi_{t}\left(z^{\prime}\right)$; that is, $\left(\psi_{t}(z)\right)_{i} \leq\left(\psi_{t}\left(z^{\prime}\right)\right)_{i}$ for $1 \leq i \leq n$ and $\left(\psi_{t}(z)\right)_{i} \geq\left(\psi_{t}\left(z^{\prime}\right)\right)_{i}$ for $n+1 \leq i \leq 2 n$. Obviously, this means $z^{\prime} \in \tilde{R}_{-}(\infty)$. This also implies $\tilde{R}_{-}(\infty)$ is order-convex. The invariance of $\tilde{R}_{-}(\infty)$ can be obtained from the definition directly.

Since $\tilde{R}_{-}(\infty)$ is nonempty and for any $z \in \tilde{R}_{-}(\infty),[z,+\infty)_{K} \cap \operatorname{Int} \mathbb{R}_{+}^{2 n} \subset \tilde{R}_{-}(\infty)$, $\tilde{R}_{-}(\infty)$ has nonempty interior. This also means $R_{-}(\infty)$ is nonempty. If $z \in R_{-}(\infty)$, then there is a neighborhood $U$ of $z$ with $U \subset R_{-}(\infty)$. In particular, there is some $z^{\prime} \ll_{K} z$ with $z^{\prime} \in U \subset R_{-}(\infty)$ and hence $\left[z^{\prime},+\infty\right)_{K} \subset \tilde{R}_{-}(\infty)$. Therefore $[z,+\infty)_{K} \subset$ $\left(z^{\prime},+\infty\right)_{K} \subset$ the interior of $\tilde{R}_{-}(\infty)=R_{-}(\infty)$. The invariance of $R_{-}(\infty)$ comes from the fact that the interior of an invariant set is also invariant.

Similar to the proof of (d) of Theorem 2.1, to prove the $V_{-}(\infty)$ is unordered, suppose that there are $z, z^{\prime} \in V_{-}(\infty)$ with $z<_{K} z^{\prime}$. Since $z, z^{\prime} \in \operatorname{Int} \mathbb{R}_{+}^{2 n}, \psi_{t}(z) \ll_{K} \psi_{t}\left(z^{\prime}\right)$ for $t<0$ by the strong backward monotonicity. Without loss of generality, assume $z \ll_{K} z^{\prime}$. There are $z_{k}, z_{k}^{\prime} \in R_{-}(\infty)$ such that $z_{k} \gg_{K} z, z_{k}^{\prime} \gg_{K} z^{\prime}$ for $k=1,2, \cdots$ and $z_{k} \rightarrow z, z_{k}^{\prime} \rightarrow z^{\prime}$ as $k \rightarrow+\infty$. Hence it is easy to find a sufficiently large integer $k$ such that $z_{k}<z^{\prime}<z_{k}^{\prime}$ and then $z^{\prime} \in R_{-}(\infty)$. But $R_{-}(\infty)$ is open and then $R_{-}(\infty) \cap V_{-}(\infty)=\emptyset$. This is a contradiction.

Last, we prove the invariance of $V_{-}(\infty)$. Suppose that $z \in V_{-}(\infty)$. For any $t>0$ it is obvious that $T_{\psi_{t}(z)}<-t$ and hence for any point $z^{\prime}$ sufficiently close to $\psi_{t}(z), T_{z^{\prime}}<-t$ too. Suppose that $z_{k} \rightarrow \psi_{t}(z)$ as $k \rightarrow+\infty$ and $z_{k} \gg_{K} \psi_{t}(z)$. Then $\psi_{-t}\left(z_{k}\right) \gg_{K} z$ and $\psi_{-t}\left(z_{k}\right) \rightarrow z$. This means that $\psi_{-t}\left(z_{k}\right) \in(z,+\infty)_{K} \cap \operatorname{Int} \mathbb{R}_{+}^{2 n} \subset R_{-}(\infty)$ and hence $z_{k} \in R_{-}(\infty)$ for any sufficiently large integer $k$ since $R_{-}(\infty)$ is invariant. Furthermore, we show that there is no sequence $\left\{z_{k}\right\} \subset R_{-}(\infty), z_{k} \rightarrow \psi_{t}(z)$ as $k \rightarrow+\infty$ and $z_{k} \ll_{K} \psi_{t}(z)$. Otherwise, the strong backward monotonicity implies $\psi_{-t}\left(z_{k}\right) \ll_{K} z$ and $\psi_{-t}\left(z_{k}\right) \rightarrow z$. But $z \in V_{-}(\infty)$, a contradiction. Hence, $V_{-}(\infty)$ is invariant.

The proofs for $\tilde{R}_{+}(\infty), R_{+}(\infty)$, and $V_{+}(\infty)$ are similar.

Proof of Proposition 2.4. We can prove this proposition just as we did for 2n-dimensional type-K competitive Kolmogorov systems in $[9$.

Let $\bar{z} \in \tilde{R}_{-}(\infty)$ and $\tilde{z} \in \tilde{R}_{+}(\infty)$. For any $z=\left(z_{1}, \cdots, z_{2 n}\right) \in \operatorname{Int} \mathbb{R}_{+}^{2 n}$, define $Z=\left(Z_{1}, \cdots, Z_{2 n}\right)$ with $Z_{i}=\ln z_{i}$. Then $H: \operatorname{Int} \mathbb{R}_{+}^{2 n} \rightarrow \mathbb{R}^{2 n}$ with $H(z)=Z$ is 
a homeomorphism from $\operatorname{Int} \mathbb{R}_{+}^{2 n}$ to $\mathbb{R}^{2 n}$. Obviously, $H\left(R_{-}(\infty)\right)$ is open in $\mathbb{R}^{2 n}$, and $Z \in H\left(R_{-}(\infty)\right)$ for any $Z>_{K} H(\bar{z})$.

Let $u$ be a vector in Int $K, E \subset \mathbb{R}^{2 n}$ be its orthogonal hyperplane, and $\mathcal{H}$ be the closed halfspace of $\mathbb{R}^{2 n}$ comprising all vectors with the form $y+\lambda u, y \in E, \lambda \geq 0$. For each $y \in E$ denote by $\mu_{y}$ the infimum of the number $\mu$ such that $y+\mu u \in H\left(R_{-}(\infty)\right)$, which exists because for any sufficiently large positive number $\mu, y+\mu u>_{K} H(\bar{z})$, and for a sufficiently small negative number $\mu, y+\mu u<_{K} H(\tilde{z})$, where we use the fact that $\tilde{R}_{-}(\infty)$ and $\tilde{R}_{+}(\infty)$ are disjoint. It is easy to see that we obtain a homeomorphism $h: \mathcal{H} \rightarrow \operatorname{clos} H\left(R_{-}(\infty)\right)$ by defining $h(y+\lambda u)=y+\lambda u+\mu_{y} u$.

It follows from $h(E)=H\left(V_{-}(\infty)\right)$ that $H\left(V_{-}(\infty)\right)$ is homeomorphic to $\mathbb{R}^{2 n-1}$, and so is $V_{-}(\infty)$. Since $h(\mathcal{H} \backslash E)=H\left(R_{-}(\infty)\right)$, it follows that $H\left(R_{-}(\infty)\right)$ is homeomorphic to $\mathbb{R}^{2 n}$, and so is $R_{-}(\infty)$. The closeness of $V_{-}(\infty)$ can be deduced from the homeomorphic properties of $h$ and $H$ easily. This completes the proof of the proposition for $R_{-}(\infty)$ and $V_{-}(\infty)$. The proof for the case of $R_{+}(\infty)$ and $V_{+}(\infty)$ is similar.

Proof of Proposition 2.5. First, we recall that the flow of the type-K competitive system in Theorem 2.1] is backward type-K monotone and strongly backward type-K monotone Int $\mathbb{R}_{+}^{2 n}$. Then $\psi_{t}(a) \ll_{K} \psi_{t}(b)$ for $t<0$. Without loss of generality, suppose $\alpha(z) \subset \operatorname{Int} \mathbb{R}_{+}^{2 n}$.

The proof can be divided into the following two cases:

(i) $\alpha(z) \not \subset \alpha\left(z^{\prime}\right)$;

(ii) $\alpha(z) \subset \alpha\left(z^{\prime}\right)$.

Case (i). Let $a \in \alpha(z) \subset \operatorname{Int} \mathbb{R}_{+}^{2 n}$, but $a \notin \alpha\left(z^{\prime}\right)$. Then there is some $b \in \alpha\left(z^{\prime}\right)$ such that $a<_{K} b$. By the strong backward monotonicity of the system, we may assume that $a \ll_{K} b$. Define

$$
\zeta(a, b)=\inf \left\{r \in \mathbb{R}: a<_{K} \psi_{t}(b), t \in[r, 0]\right\} .
$$

By the result of Hirsch (see [2], 12]), $\zeta(a, b)=-\infty$; that is, $a<_{K} \psi_{t}(b)$ for all $t \leq 0$. Hence, $a \leq_{K} \alpha(b)$. But $a \notin \alpha(b) \subset \alpha\left(z^{\prime}\right)$. So $a<_{K} \alpha(b)$. Since $\alpha(b)$ is invariant and $a \in \operatorname{Int} \mathbb{R}_{+}^{2 n}, \psi_{-1}(a) \ll_{K} \alpha(b)$. Therefore for some $t<0, \psi_{t}(z) \ll_{K} \alpha(b)$. Using the strong backward monotonicity and the invariance of $\alpha(b)$ again, we get that $\alpha(z) \leq_{K}$ $\alpha(b)$. We claim that there is some $c \in \alpha(b) \subset \alpha\left(z^{\prime}\right)$ such that $\alpha(z)<_{K} c$. Otherwise, $\alpha(b) \subset \alpha(z)$. If there is some $d \in \alpha(z)$ with $d \notin \alpha(b)$, then $d<_{K} \alpha(b)$, contradicting that $\alpha(z)$ is unordered in the type-K order. Hence $\alpha(z)=\alpha(b) \subset \alpha\left(z^{\prime}\right)$, contradicting our assumption. Our claim holds. Furthermore, $\alpha(z) \subset \operatorname{Int} \mathbb{R}_{+}^{2 n}$. By the strong backward type-K monotonicity of the system, let $\alpha(z) \ll_{K} c$ without loss of generality. This implies there is some $t \in \mathbb{R}$ such that $\alpha(z) \ll_{K} \psi_{t}\left(z^{\prime}\right)$ and $\alpha(z) \leq_{K} \alpha\left(z^{\prime}\right)$. If there is some point $d \in \alpha(z) \cap \alpha\left(z^{\prime}\right)$, then $\alpha(z) \leq_{K} d \leq_{K} \alpha\left(z^{\prime}\right)$. But the nonordering of $\alpha(z)$ and $\alpha\left(z^{\prime}\right)$ implies that $\alpha(z)=d=\alpha\left(z^{\prime}\right)$, a contradiction. Therefore, $\alpha(z)<_{K} \alpha\left(z^{\prime}\right)$. Moreover, the strong backward type-K monotonicity of the system and the invariance of $\alpha(z)$ and $\alpha\left(z^{\prime}\right)$ imply $\alpha(z) \ll_{K} \alpha\left(z^{\prime}\right)$.

Case (ii). We want to prove $\alpha(z)=\alpha\left(z^{\prime}\right)$. In fact, if there is some $c \in \alpha\left(z^{\prime}\right)$ but $c \notin \alpha(z)$, then there is some $d \in \alpha(z)$ with $d<_{K} c$. But $d \in \alpha(y) \cap \operatorname{Int} \mathbb{R}_{+}^{2 n}$, contradicting the nonordering of the $\alpha$-limit set. Hence $\alpha(z)=\alpha\left(z^{\prime}\right)$. Moreover, $\alpha(z)=\alpha\left(z^{\prime}\right) \subset \mathcal{E}$ (see [2] or [12]).

The last conclusion is obvious. 
Proof of Theorem 2.6. We divide the proof into the following four parts.

Part 1. We want to prove that if $\alpha(z) \subset \operatorname{Int} \mathbb{R}_{+}^{2 n}$ for some $z \in \operatorname{Int} \mathbb{R}_{+}^{2 n}$, then every neighborhood of $z$ contains two points $z^{1}, z^{2}$ with $z^{2}<_{K} z<_{K} z^{1}$ such that either $z^{1} \in R_{-}(\infty)$ or $\alpha\left(z^{1}\right) \subset \mathcal{E}$, and either $z^{2} \in R_{+}(\infty)$ or $\alpha\left(z^{2}\right) \subset \mathcal{E}$.

Only consider $z^{1} ; z^{2}$ is similar. We check the following two cases:

(i) $(z,+\infty)_{K} \cap \Gamma=\emptyset$;

(ii) there is some $z^{\prime}>_{K} z$ such that $\left[z, z^{\prime}\right]_{K} \subset \Gamma$.

Case (i). We claim that $(z,+\infty)_{K} \cap \operatorname{Int} \mathbb{R}_{+}^{2 n} \subset R_{-}(\infty)$. In fact, for any $z^{\prime} \in$ $(z,+\infty)_{K} \cap \operatorname{Int} \mathbb{R}_{+}^{2 n}$ and $t \in\left(T_{z^{\prime}}, 0\right), \psi_{t}(z) \ll_{K} \psi_{t}\left(z^{\prime}\right)$. This means that $\left(\psi_{t}\left(z^{\prime}\right)\right)_{i}>$ $\left(\psi_{t}(z)\right)_{i}$ for $i=1, \cdots, n$ and $\left(\psi_{t}\left(z^{\prime}\right)\right)_{i}<\left(\psi_{t}(z)\right)_{i}$ for $i=n+1, \cdots, 2 n$. Moreover, we have $\inf \left\{\left(\psi_{t}\left(z^{\prime}\right)\right)_{i}, t \in\left(T_{z^{\prime}}, 0\right)\right\}>0$ for $i=1, \cdots, n$ and $\sup \left\{\left(\psi_{t}\left(z^{\prime}\right)\right)_{i}, t \in\left(T_{z^{\prime}}, 0\right)\right\}<+\infty$ for $i=n+1, \cdots, 2 n$. Hence $z^{\prime} \in R_{-}(\infty)$.

Case(ii). Using our Proposition 2.5] and the result in [2] for the type-K order interval $\left[z, z^{\prime}\right]_{K}$, we can obtain that every neighborhood of $z$ contains a point $z^{1}$ with $z \leq_{K} z^{1}$ such that $\alpha\left(z^{1}\right) \subset \mathcal{E}$.

Part 2. In this part, we want to prove that for any point $z \in \mathcal{K}$, any neighborhood of $z$ contains two points $z^{1}, z^{2}$ with $z^{2} \ll_{K} z \ll_{K} z^{1}$ such that either $z^{1} \in R_{-}(\infty)$ or $\alpha\left(z^{1}\right)$ contains an equilibrium $\geq_{K} z$ and either $z^{2} \in R_{+}(\infty)$ or $\alpha\left(z^{2}\right)$ contains an equilibrium $\leq_{K} z$.

We still only consider $z^{1}$. Fix $z \in \mathcal{K}$. Then $\psi_{1}(z) \in \mathcal{K}$ by the invariance of $\mathcal{K}$. Applying the conclusion of Part 1 to $\psi_{1}(z)$, we obtain that there exists some $w^{1}>_{K} \psi_{1}(z)$ such that either $w^{1} \in R_{-}(\infty)$ or $\alpha\left(w^{1}\right) \subset \mathcal{E}$. Then, by the type-K monotonicity of $\psi_{-1}$, we have $z \ll_{K} \psi_{-1}\left(w^{1}\right):=z^{0}$ and either $z^{0} \in R_{-}(\infty)$ or $\alpha\left(z^{0}\right) \subset \mathcal{E}$. Because $z$ is in the limit set $\mathcal{K}, z$ is nonwandering; that is, there are convergent sequences $z^{i} \rightarrow z$ and $t_{i} \rightarrow-\infty$ in $\mathbb{R}$ such that $\psi_{t_{i}}\left(z^{i}\right) \rightarrow z$, and we can take $t_{i}<0$ and $z^{i} \ll_{K} z^{0}$. Passing to a subsequence, we assume $\psi_{t_{i}}\left(z^{0}\right)$ converges as $i \rightarrow \infty$ to a point $q \in \alpha\left(z^{0}\right) \subset \mathcal{E}$. Since the flow $\psi$ is strongly backward type-K monotone in $\operatorname{Int} \mathbb{R}_{+}^{2 n}, \psi_{t_{i}}\left(z^{i}\right) \ll_{K} \psi_{t_{i}}\left(z^{0}\right)$. Therefore $z \leq_{K} q$.

Part 3. In this part, we want to prove that if $q$ is an equilibrium and $z<_{K} q$ for some $z \in \mathcal{K}$, then $\mathcal{K} \ll_{K} q$.

By the strong backward type-K monotonicity, $\psi_{t}(z) \ll_{K} q$ for $t<0$, so there are points of $\mathcal{K}$ that are $\ll_{K} q$. Suppose that $\mathcal{K}=\omega(v)$. It follows that for any $t \in \mathbb{R}$ there exists some $s>t$ such that $\psi_{s}(v) \ll_{K} q$. Therefore $\psi_{t}(v) \ll_{K} q$ for all $t \in \mathbb{R}$ by the strong backward type-K monotonicity. Thus $\omega(v) \leq_{K} q$. In fact $\omega(v)<_{K} q$, since $q \in \omega(v)$ would imply $\mathcal{K}=q$; otherwise $\mathcal{K}$ would contain two points related by $<_{K}$, contradicting the nonordering of $\mathcal{K}$. Therefore, by the invariance of $\mathcal{K}$ and $q$ and the strong backward type-K monotonicity, $\omega(v) \ll_{K} q$. A similar argument applies if $\mathcal{K}=\alpha(v)$.

Part 4. Now, we complete the proof of the theorem.

Applying the conclusion of Part 2, we get that for any $z \in \mathcal{K}$, its every neighborhood contains a point $v_{z} \gg_{K} z$ such that either $v_{z} \in R_{-}(\infty)$ or $\alpha\left(v_{z}\right)$ contains an equilibrium $q_{z} \geq_{K} z$. If for any $z \in \mathcal{K}$ and any neighborhood of $z, v_{z} \in R_{-}(\infty)$, then the theorem obviously holds. In this case $\mathcal{K} \subset V_{-}(\infty)$. Otherwise, for some $z \in \mathcal{K}$ and some neighborhood of $z, v_{z} \gg_{K} z$ and $q_{z} \geq_{K} z$ such that $q_{z} \in \alpha\left(v_{z}\right) \subset \mathcal{E}$ by the conclusion of Part 2. We claim that $z<_{K} q_{z}$. Suppose not, i.e., $q_{z}=z \ll_{K} v_{z}$. Then $\alpha\left(v_{z}\right)=q_{z}=z$. 
But $z \in \mathcal{K}$ is not an isolated point in $\mathcal{K}$. There is some $z^{\prime} \in \mathcal{K}$ with $z^{\prime} \neq z$ satisfying $z^{\prime} \ll_{K} v_{z}$. Since $z^{\prime} \in \mathcal{K}, z^{\prime}$ is nonwandering. Thus there are convergent sequences $z^{i} \rightarrow z^{\prime}$ and $t_{i} \rightarrow-\infty$ in $\mathbb{R}$ such that $\psi_{t_{i}}\left(z^{i}\right) \rightarrow z^{\prime}$, and we can take $t_{i}<0$ and $z^{i} \ll_{K} v_{z}$. By the strong type-K monotonicity, we have $\psi_{t_{i}}\left(z^{i}\right) \ll_{K} \psi_{t_{i}}\left(v_{z}\right)$. Letting $i \rightarrow \infty$, we obtain that $z^{\prime} \leq_{K} z$. The nonordering of $\mathcal{K}$ implies $z=z^{\prime}$, a contradiction. Our claim holds. By the conclusion of Part $3, \mathcal{K} \ll_{K} q_{z}$. But we cannot find a point $a$ with $\mathcal{K} \ll_{K} a \ll_{K} q_{z}$ and $a \in R_{-}(\infty)$. Hence, for any $z \in \mathcal{K}$ and any neighborhood $U_{z}$ of $z$, we can pick $v_{z} \in U_{z}$ with $v_{z} \gg_{K} z$ and $q_{z}>_{K} z$ with $q_{z} \in \alpha\left(v_{z}\right) \subset \mathcal{E}$.

It now follows from Part 3 that $\mathcal{K} \ll_{K} q_{z}$ for every $z \in \mathcal{K}$. Let $q \in \mathcal{E}$ be the greatest lower bound of the set of equilibria $\gg_{K} \mathcal{K}$ in the type-K order. Then $q \geq_{K} \mathcal{K}$. In fact $q \notin \mathcal{K}$. Otherwise $\mathcal{K}$ would equal $q$, contradicting the hypothesis. Moreover, by the invariance of $q$ and $\mathcal{K}$ and the strong backward type-K monotonicity, $q \gg_{K} \mathcal{K}$.

By choosing $v_{z} \ll_{K} q$, we see that $q_{z} \leq_{K} q$ and therefore $q_{z}=q$ for all $z \in \mathcal{K}$, which implies that $\alpha\left(v_{z}\right)=q$ for every $z \in \mathcal{K}$. Since $v_{z}$ can also be taken arbitrarily near $z$, it follows that $\mathcal{K} \subset V_{-}(q)$.

Similarly we can prove the case that $\mathcal{K}$ is contained in the upper repulsion boundary of some $R_{+}(p)$ for $p \in \mathcal{E} \cup\{\infty\}$.

Proof of Theorem 2.8. Suppose that $z \notin V_{-}(q)$. Otherwise the theorem obviously holds. Here $\omega(z) \subset V_{-}(q) \cap \operatorname{Int} \mathbb{R}_{+}^{n}$ implies that the distance between $\psi_{t}(z)$ and $V_{-}(q)$ tends to 0 as $t \rightarrow \infty$. It is easy to see that $\left[\psi_{t}(z),+\infty\right)_{K} \cap V_{-}(q)$ and $\left(-\infty, \psi_{t}(z)\right]_{K} \cap V_{-}(q)$ are compact in $\mathbb{R}_{+}^{2 n}$ and just one of them is nonempty for any sufficiently large $t$. Without loss of generality, we may suppose that $\left[\psi_{t_{n}}(z),+\infty\right)_{K} \cap V_{-}(q)$ is nonempty for $t_{n} \rightarrow+\infty$. We also know that if $\left[\psi_{t}(z),+\infty\right)_{K} \cap V_{-}(q)$ is nonempty, then by the backward type-K monotonicity of $\psi$ and the invariance of $V_{-}(q),\left[\psi_{s}(z),+\infty\right)_{K} \cap V_{-}(q)$ is nonempty for all $s \in\left(T_{z}, t\right]$. The above two arguments show that $\left[\psi_{t}(z),+\infty\right)_{K} \cap V_{-}(q)$ is nonempty for all $t \in\left(T_{z},+\infty\right)$. For any $t>0, \psi_{-t}\left(\left[\psi_{t}(z),+\infty\right)_{K} \cap V_{-}(q)\right) \subset[z,+\infty)_{K} \cap V_{-}(q)$ since $\psi_{-t}$ is a type-K monotone map and $V_{-}(q)$ is invariant. $\bigcap_{t \geq 0} \psi_{-t}\left(\left[\psi_{t}(z),+\infty\right)_{K} \cap V_{-}(q)\right)$ is nonempty. Hence, there exists some $z^{\prime} \in V_{-}(q)$ such that $\psi_{t}(z) \leq_{K} \psi_{t}\left(z^{\prime}\right)$ for any $t \in \mathbb{R}^{+}$. Furthermore, this type-K order relation holds for all $t \in\left(T_{x},+\infty\right)$ by the backward type-K monotonicity.

To see that $\left\|\psi_{t}\left(z^{\prime}\right)-\psi_{t}(z)\right\| \rightarrow 0$ as $t \rightarrow \infty$, suppose that this is not so. Then there is a sequence $s_{j} \rightarrow \infty$ such that $\psi_{s_{j}}(z) \rightarrow a \in \omega(z) \subset V_{-}(q)$ and $\psi_{s_{j}}\left(z^{\prime}\right) \rightarrow b \in V_{-}(q)$. Then necessarily $a<_{K} b$, contradicting (d) of Theorem 2.1.

Proof of Corollary 2.9. In this case, for any equilibrium $z$, the trace of the Jacobian matrix $D F(z)$ of the vector field at $z$ is less than 0 . Hence $D F(z)$ has an eigenvalue $s<0$. This means $z$ cannot repulse any open set and the sets $R_{-}(z)$ and $R_{+}(z)$ are empty. Hence, the conclusions of this corollary can be obtained by Theorems 2.6 and 2.8 directly.

\section{REFERENCES}

[1] M. Hirsch, Systems of differential equations which are competitive or cooperative. I: Limit sets, SIAM J. Math. Anal., 13 (1982), 167-179. MR0647119 (83i:58081)

[2] M. Hirsch, Systems of differential equations which are competitive or cooperative. II: Convergence almost everywhere, SIAM J. Math. Anal., 16 (1985), 423-439. MR0783970(87a:58137) 
[3] M. Hirsch, Systems of differential equations which are competitive or cooperative. III: Competing species, Nonlinearity, 1 (1988), 51-71. MR0928948(90d:58070)

[4] S. B. Hsu, H. L. Smith, and P. Waltman, Competitive exclusion and coexistence for competitive systems on ordered Banach spaces, Trans. Amer. Math. Soc., 348(1996), 4083-4094. MR1373638 (97d:92021)

[5] E. Kamke, Zur Theorie der Systeme Gewoknlicher Differentialiechungen, II, Acta Math. 58(1932), $57-85$.

[6] X. Liang and J. Jiang, On the finite dimensional dynamical systems with limited competition, Trans. Amer. Math. Soc., 354 (2002), 3535-3554. MR1911510 (2003d:37149)

[7] X. Liang and J. Jiang, The classification of the dynamical behavior of 3-dimensional type- $K$ competitive Lotka-Volterra systems, Nonlinear Analysis, TMA, 51:5(2002), 749-763. MR1921374 (2003e:37133)

[8] X. Liang and J. Jiang, Discrete infinite dimensional type-K monotone dynamical systems and time periodic reaction-diffusion systems, J. Differential Equation, 189:1(2003), 318-354. MR1968324 (2004b:37176)

[9] X. Liang and J. Jiang, The dynamical behavior of type-K competitive Kolmogorov systems and its applications to 3-dimensional type-K competitive Lotka-Volterra systems, Nonlinearity, 16:3(2003), 785-801. MR $1975782(2004 \mathrm{~m}: 37167)$

[10] J. Murray, Mathematical Biology, 1989, Springer-Verlag, Berlin. MR.1007836 (90g:92001)

[11] S. Smale, On the differential equations species in competition, J. Math. Biol., 3(1976), 5-7. MR0406579 (53:10366)

[12] H. L. Smith, "Monotone Dynamical Systems, an Introduction to the Theory of Competitive and Cooperative Systems," Mathematical Surveys and Monographs, vol. 41(1995), Amer. Math. Soc., Providence, RI. MR 1319817 (96c:34002)

[13] H. L. Smith, Competing subcommunities of mutualists and a generalized Kamke theorem, SIAM J. Appl. Math., 46(1986), 856-874. MR0858998 (87i:92047)

[14] H. L. Smith and H. R. Thieme, Stable coexistence and bi-stability for competitive systems on ordered Banach spaces, J. Diff. Eqns., 176 (2001), no. 1, 195-222. MR1861187(2002i:47084)

[15] P. Takáč, Convergence to equilibrium on invariant d-hypersurfaces for strongly increasing discretetime semigroups, J. Math. Anal. Appl. 148 (1990), 223-244. MR.1052057 (91d:58125)

[16] Y. Takeuchi, Diffusion-mediated persistence in two-species competition Lotka-Volterra model, Math. Biosciences 95(1989), 65-83. MR1001292 (90e:92073)

[17] Y. Takeuchi and Z. Lu, Permanence and global stability for competitive Lotka-Volterra diffusion systems, Nonlinear Analysis, TMA, 24(1995), 91-104. MR1308472

[18] C. F. Tu and J. F. Jiang, The coexistence of a community of species with limited competition, J. Math. Anal. Appl., 217(1998), 553-571. MR1492087 (99c:92061)

[19] C. F. Tu and J. F. Jiang, The necessary and sufficient conditions for the global stability of type-K Lotka-Volterra systems, Proc. Amer. Math. Soc., 127(1999), 3181-3186. MR.1628420|(2000b:34082)

[20] C. F. Tu and J. F. Jiang, Global stability and permanence for a class of type-K monotone systems, SIAM J. Math. Anal., 30(1999), 360-378. MR.1664764 (99j:34073)

[21] Y. Wang and J. Jiang, The long-run behavior of periodic competitive Kolmogorov systems, Nonlinear Anal. Real World Appl., 3(2002), 471-485. MR1930616 (2003g:34112)

[22] Y. Wang and J. Jiang, Uniqueness and attractivity of the carrying simples for the discretetime competitive dynamical systems, J. Differential Equations, 186(2002), 611-632. MR1942224 (2003k:34083)

[23] M. L. Zeeman, Hopf bifurations in competitive three-dimensional Lotka-Volterra systems, Dynamics and Stability of Systems, 3(1993), 190-217. MR1246002 (94j:34044) 